\title{
Expression of FoxM1 and the EMT-associated protein E-cadherin in gastric cancer and its clinical significance
}

\author{
JING ZHANG* ${ }^{*}$, XIAO-YU CHEN*, KE-JIAN HUANG* , WEI-DONG WU, TAO JIANG, \\ JUN CAO, LI-SHENG ZHOU, ZHENG-JUN QIU and CHEN HUANG
}

Department of General Surgery, Shanghai Jiaotong University Affiliated First People's Hospital, Shanghai 200080, P.R. China

Received May 27, 2015; Accepted July 4, 2016

DOI: $10.3892 / \mathrm{ol} .2016 .4917$

\begin{abstract}
The aim of the present study was to investigate the expression of forkhead box M1 (FoxM1) and E-cadherin in tissues of gastric cancer in order to reveal any correlation between FoxM1, E-cadherin and clinicopathological parameters. The association between FoxM1 and E-cadherin in the development and progression of gastric cancer was also investigated. The expression of FoxM1 and E-cadherin in gastric cancer and adjacent normal tissue on tissue microarray was detected using immunohistochemistry. The clinicopathological significance of FoxM1 and E-cadherin in gastric cancer was explored, and the association between FoxM1 and E-cadherin was further examined using statistical techniques. In gastric cancer tissues, the expression of FoxM1 and E-cadherin was strongly positive, but it was weak in normal gastric mucosa. Overexpression of FoxM1 was evident in gastric cancer, and was associated with poor tumor differentiation $(\mathrm{P}<0.05)$, advanced tumor state $(\mathrm{P}<0.05)$ and lymph node (or distant) metastasis $(\mathrm{P}<0.05)$, whereas E-cadherin had the opposite effects. Furthermore, the correlation between FoxM1 and E-cadherin expression in gastric cancer tissue was negative. In conclusion, the high FoxM1 expression and low E-cadherin expression in gastric cancer tissue suggests that these proteins play a critical role in the development and progression of gastric cancer.
\end{abstract}

\section{Introduction}

Gastric cancer, one of the leading causes of cancer-associated mortalities worldwide, is the fourth and the fifth most common cancer in males and females, respectively (1). In 2012, Nagini

Correspondence to: Dr Chen Huang or Dr Zheng-Jun Qiu, Department of General Surgery, Shanghai Jiaotong University Affiliated First People's Hospital, 100 Haining Road, Shanghai 200080, P.R. China

E-mail: richard-hc@hotmail.com

E-mail: zhengjunqiu283@126.com

*Contributed equally

Key words: FoxM1, E-cadherin, gastric cancer suggested that, in spite of the advances in diagnosis and therapeutic methods, numerous patients with gastric cancer succumb to invasion and metastasis, with only a $<20 \% 5$-year survival rate for advanced gastric cancer (2). The diverse risk factors that contribute to the development of gastric cancer include, among others, precancerous lesions, genetic factors and Helicobacter pylori infection (3). However, the reasons for the very poor prognosis of advanced gastric cancer are currently not understood. Therefore, the mechanisms involved in gastric cancer metastasis require to be urgently investigated.

Forkhead box M1 (FoxM1), a transcription factor characterized by a conserved winged-helix DNA-binding domain, was previously known as forkheaddrosophila homolog like 16, HNF-3/fork-head homolog 11, Trident and membrane palmitoylated protein 2 in the literature (4). Emerging data suggest that FoxM1 is considered to be a key regulator in the cell cycle at the G1-S and G2-M phases, by controlling the expression of essential genes and the progression of mitosis (5). Recently, numerous studies on FoxM1 have suggested that FoxM1 is implicated in angiogenesis, invasion and metastasis, indicating that FoxM1 may be oncogenic and play a significant role in tumorigenesis $(6,7)$. Based on the published literature, the expression of FoxM1 in multiple cancers is strongly positive, and markedly contributes to the progression of cancers such as pancreatic, colorectal and breast cancer (6-8). Notably, the study by Li et al reports that the expression of FoxM1 in tissue and cell lines of gastric cancer is higher than that in normal tissues, and is a favorable biomarker for the prognosis of patients with gastric cancer (9). Furthermore, the above study offers prominent evidence that enforced expression of FoxM1 in mice enhanced the tumorigenic and metastatic abilities of human gastric cancer cells, whereas attenuated FoxM1 expression did the opposite, indicating that FoxM1 occupies an important position in the progression of gastric cancer (10). Additionally, it has been reported that the expression of vascular endothelial growth factor is directly regulated by FoxM1 at the level of transcription, contributing critically to the angiogenesis of gastric cancer (10). Furthermore, it has been previously demonstrated that reduced FoxM1 expression inhibits the ability of proliferation and invasion of nasopharyngeal carcinoma cells by modulating the epithelial-to-mesenchymal transition (EMT) (11). In contrast, elevated expression of FoxM1 increases the invasion of hepatocellular carcinoma cells by suppressing the expression of epithelial cell markers 
and acquiring the EMT phenotype (12). Due to the critical role of FoxM1 in multiple tumors, it is essential to determine the precise mechanisms and functions of FoxM1 in tumors, particularly in gastric cancer, which is rarely studied.

E-cadherin, also termed cadherin 1 , is a pivotal membrane-spanning glycoprotein of epithelial cells, containing an extracellular structure consisting of five cadherin repeats (13). Accumulating evidence has demonstrated that E-cadherin functions in several aspects of cellular activity, including cell communication, cell signalling, cell recognition, tissue morphogenesis, oncogenesis and, in particular, intercellular adhesion (13). Notably, little is known about the role of E-cadherin in EMT, which induces the invasion and metastasis of cancer cells (14). Yu et al have verified that a reduction in E-cadherin expression is associated with the loss of epithelial phenotypes, which play a critical role in the process of EMT (15). Furthermore, patients with loss of E-cadherin in breast cancer have a higher proportion of mesenchymal phenotype tumor cells circulating compared with patients with normal E-cadherin expression, demonstrating that E-cadherin may also be responsible for the diffusion of circulating tumor cells with a mesenchymal phenotype (16). Despite the large body of research on the role of E-caherin in the development of EMT, the underlying regulatory mechanism(s) of E-caherin remain(s) largely unknown, particularly in the case of gastric cancer.

The present study aims to clarify the clinical significance of FoxM1 and E-cadherin and to establish any correlation between FoxM1 and E-cadherin, which may help to establish the role of FoxM1 in EMT, invasion and metastasis of gastric cancer.

\section{Materials and methods}

Tissue microarray. All gastric cancer tissue microarrays used in the present study were purchased from US Biomax, Inc. (Rockville, MD, USA), including 70 cases of gastric cancer tissues and 5 cases of normal gastric mucosa. Each core of the tissue microarray represents a sample. US Biomax, Inc. also provided patient information concerning age, gender, clinical stage, histological grade and pathological diagnosis.

Tissue immunohistochemistry. The expression of FoxM1 and E-cadherin in gastric cancer tissue microarrays was analyzed using rabbit anti-FoxM1 polyclonal antibody (cat. no. sc-500; 1:100; Santa Cruz Biotechnology, Inc., Dallas, TX, USA) and mouse anti-E-cadherin monoclonal antibody (cat. no. 610182; 1:200; BD Biosciences, Franklin Lakes, NJ, USA). Standard immunohistochemical procedures were implemented as follows: The samples on slides embedded with paraffin and formalin were firstly dewaxed in xylene and rehydrated in alcohol gradients of 100, 95, 85 and $75 \%$. The slides were then heated to $95^{\circ} \mathrm{C}$ for $30 \mathrm{~min}$ to retrieve antigens in the tissues. The activity of endogenous peroxidase was blocked by employing 3\% hydrogen peroxide for $10 \mathrm{~min}$. Part of the tissue was covered with normal serum (Proteintech Group, Inc., Rosemont, IL, USA) at room temperature for $30 \mathrm{~min}$. Then, the samples on the slides were incubated with antibodies against FoxM1 and E-cadherin overnight at $4^{\circ} \mathrm{C}$. The slides were subsequently incubated with peroxidase-conjugated AffiniPure goat anti-rabbit (cat. no. SA00001-2; 1:500) and goat ant-mouse (cat. no. SA00001-1; 1:500) Ig G (H+L)
(Jackson ImmunoResearch Laboratories, Inc., West Grove, PA, USA) for $1 \mathrm{~h}$ at room temperature. Next, the samples were stained with 3,3'-diaminobenzidine and counterstained with hematoxylin. The procedure of dehydration was implemented, and finally covers slips were applied.

Immunohistochemistry score. The staining scores were evaluated independently by two investigators, who were blinded to the information on the microarrays. Based on the staining intensity and number of positive cells, FoxM1 and E-cadherin samples were sorted into three groups, namely, a negative group, a weak positive group and a strong positive group. The staining intensity was categorized into four grades as follows: No staining, 0 ; light staining, 1; moderate staining, 2 ; and dark staining, 3 . The percentage of positive cells was classified into five groups as follows: $<10 \%, 0 ; 10-25 \%, 1 ; 25-50 \%, 2 ; 50-75 \%, 3$; and $>75 \%$, 4. The overall scores of $\leq 3,3-6$ and $>6$ were termed as negative, weakly positive and strongly positive, respectively.

Statistical analysis. The differences between FoxM1 and E-cadherin expression and clinicopathological parameters from the tissue microarray specimens were determined by the Wilcoxon rank-sum test. The correlation between FoxM1 and E-cadherin expression was determined by the Spearman's rank correlation test $(r, P) . P<0.05$ was considered to indicate a statistically significant difference. SPSS version 17.0. (SPSS, Inc., Chicago, IL, USA) was used for the analysis.

\section{Results}

Expression of FoxM1 in gastric cancer samples and its direct association with clinicopathological features. FoxM1 expression in gastric cancer was detected primarily in the cytoplasm and nuclear compartment. The results demonstrated that the staining of FoxM1 in normal gastric mucosa was negative, whereas the staining of FoxM1 in the majority of gastric cancer tissues was strong or weak positive $(\mathrm{P}<0.001$, Fig. 1A). Furthermore, the present study revealed that high expression of FoxM1 in gastric cancer was closely associated with advanced tumor stage, poor tumor differentiation and lymph node (or distant) metastasis. The expression of FoxM1 in advanced tumors (stages III and IV) was higher in comparison with that in early tumors (stages I and II) $(\mathrm{P}<0.001$, Fig. 1B). Additionally, the expression of FoxM1 in well-differentiated (grade I) and moderately-differentiated (grade II) gastric cancer was lower compared with that in poorly-differentiated (grade III) gastric cancer ( $\mathrm{P}=0.002$, Fig. $1 \mathrm{C})$. In addition, the staining of FoxM1 in samples with lymph node (or distant) metastasis was strongly positive, in contrast to that in samples with no metastasis $(\mathrm{P}<0.001$, Fig. 1D)

Expression of E-cadherin in gastric cancer samples and its direct association with clinicopathological features. The expression of E-cadherin in gastric cancer was detected mainly in the membrane of epithelial cells. The results revealed that the staining of E-cadherin in normal gastric mucosa was strongly positive, whereas the staining of E-cadherin in the majority of gastric cancer tissue samples was weakly positive or negative $(\mathrm{P}<0.001$, Fig. 2A). These results indicate that the low expression of E-cadherin in gastric cancer was closely correlated with 
A

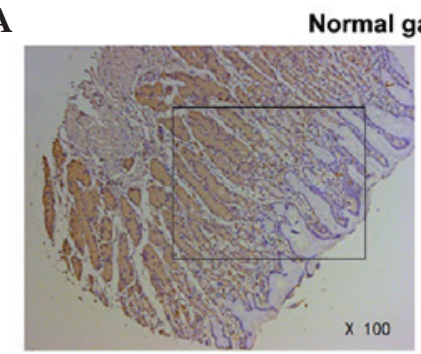

B
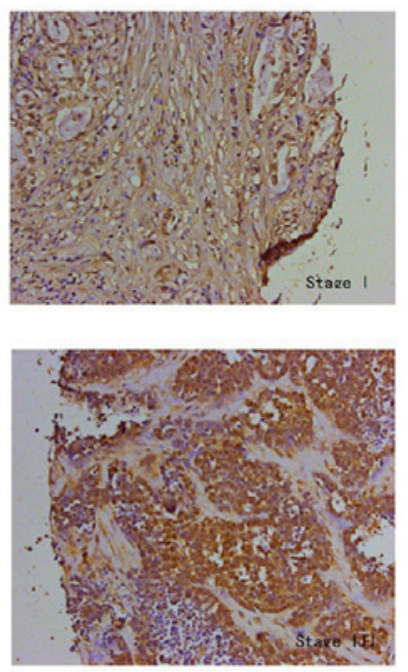

D

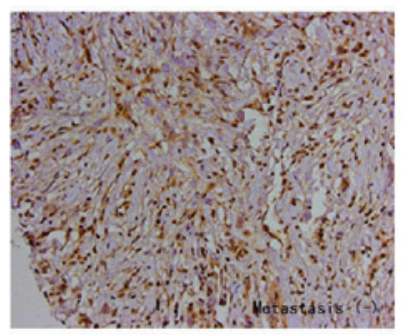

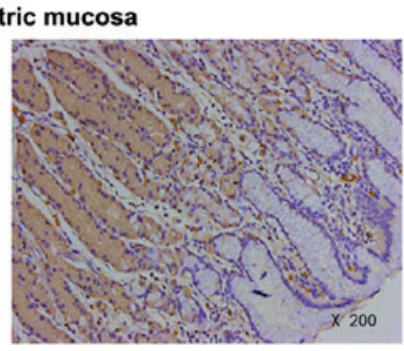

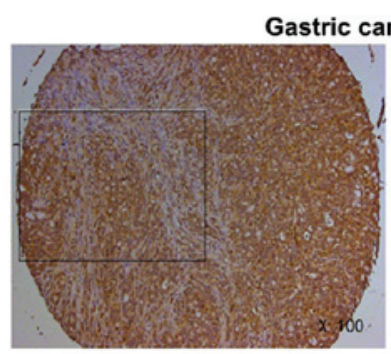

C
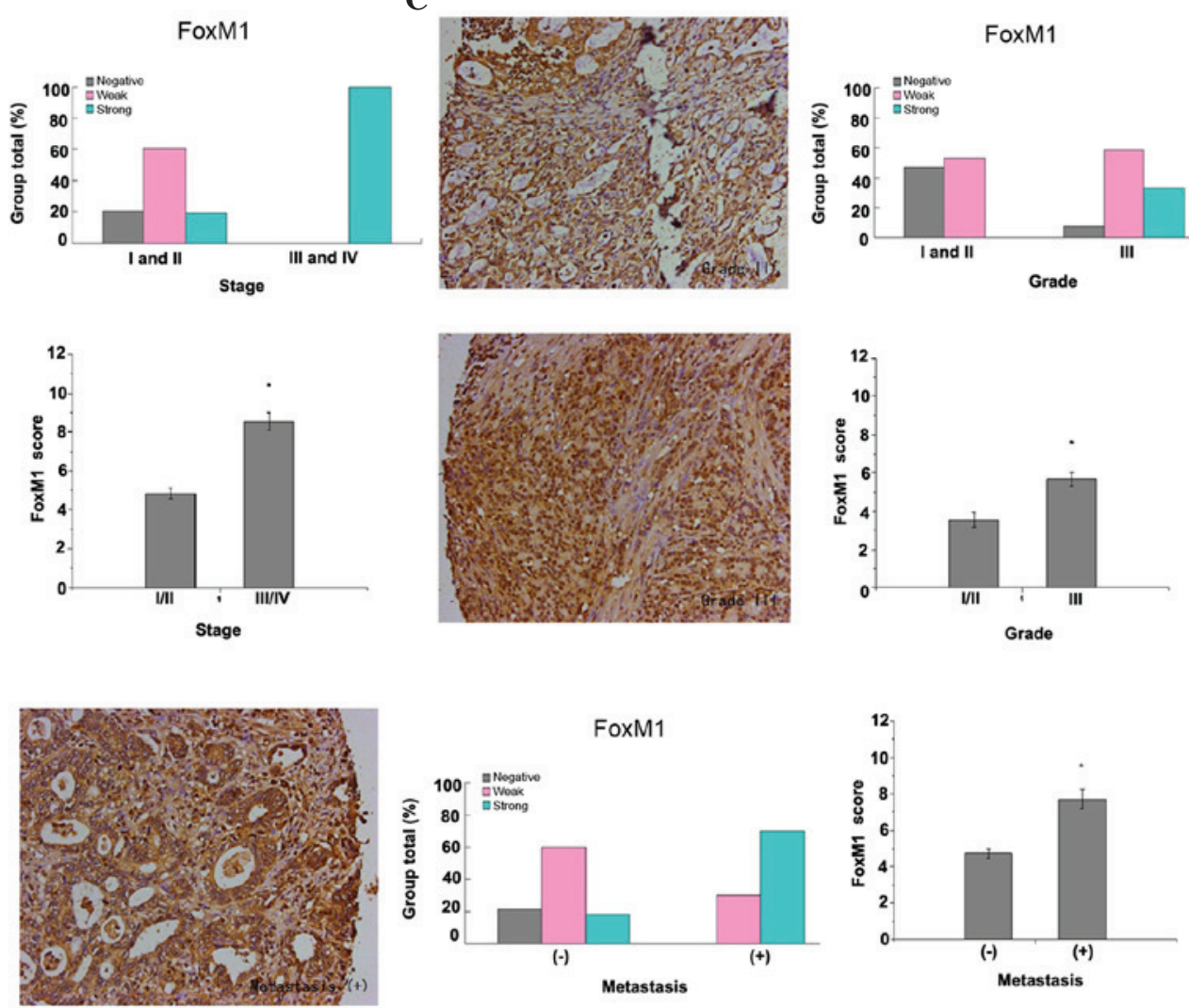

Figure 1. FoxM1 expression in samples of gastric cancer and its association with pathological features. (A) FoxM1 expression was detected by immunohistochemical analysis of tissue microarrays. Representative images of FoxM1 expression in normal gastric mucosa and gastric cancer tissue are shown (magnification, x100, and x200 for the inserts). The staining of FoxM1 in the normal gastric mucosal cells was negative, whereas the staining of FoxM1 in gastric cancer tissue cells was strongly positive. (B) FoxM1 expression was positively correlated with tumor stage (P<0.001 for stages I and II vs. stages III and IV). Representative images of stage I (weakly positive) and III (strongly positive) tumors are shown (magnification, x200). (C) FoxM1 expression was positively correlated with tumor differentiation ( $\mathrm{P}=0.002$ for grades I and II vs. grade III). Representative images of grade II and III tumors are shown (magnification, x200). (D) FoxM1 expression was positively correlated with lymph node (or distant) metastasis (P<0.001 for non-metastasis group vs. metastasis group). Representative images of gastric cancer with or without metastasis are shown (magnification, x200). FOXM1, forkhead box M1.

advanced tumor stages, poor tumor differentiation and lymph node (or distant) metastasis. The expression of E-cadherin in advanced tumors (stages III and IV) was lower in comparison with that in early tumors (stages I and II) ( $\mathrm{P}=0.029$, Fig. 2B). Additionally, the expression of E-cadherin in well-differentiated (grade I) and moderately-differentiated (grade II) gastric cancer was higher compared with that in poorly-differentiated (grade III) gastric cancer ( $\mathrm{P}=0.050$, Fig. $2 \mathrm{C})$. In addition, the expression of E-cadherin in samples that had not metastasized was strongly higher than that in samples with lymph node (or distant) metastasis ( $\mathrm{P}=0.037$, Fig. 2D)

Correlation between FoxM1 and E-cadherin expression. To clarify the association between FoxM1 and E-cadherin expression in gastric cancer, the results of immunohistochemical staining were analyzed. Among all the primary gastric cancer tissues, 36 cases with positive FoxM1 expression and negative E-cadherin expression were identified. There were 9 cases with negative FoxM1 expression and positive E-cadherin expression. The correlation between FoxM1 and E-cadherin expression in gastric cancer was negative $(\mathrm{r}=-0.255, \mathrm{P}=0.035)$. Additionally, the staining of FoxM1 in normal gastric mucosa was negative, while the staining of E-cadherin was positive in the same samples. Based on these results, a negative association appears to exist between FoxM1 and E-cadherin expression, since the staining of E-cadherin was negative in samples that were strongly positive for FoxM1 (Fig. 3), whereas the staining of E-cadherin was moderately positive in those samples that exhibited moderately- positive staining for FoxM1 (Fig. 3). 
A

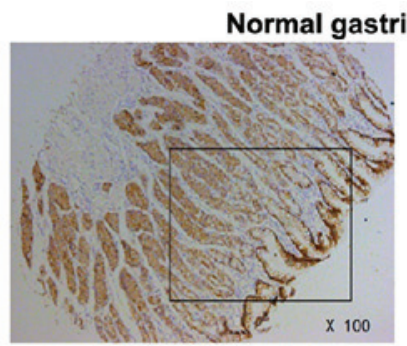

B
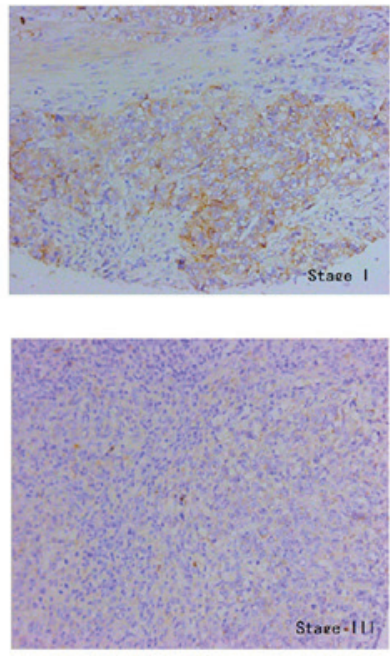

D

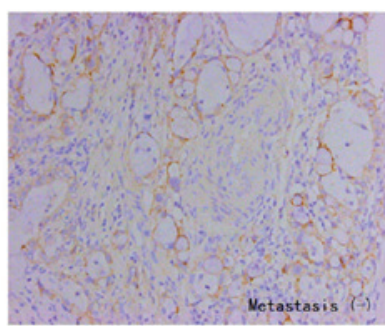

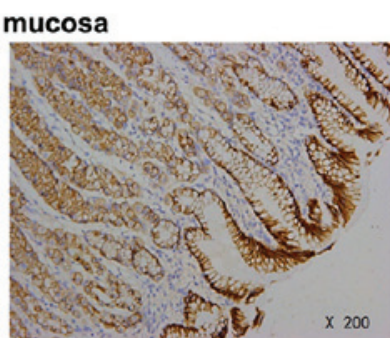

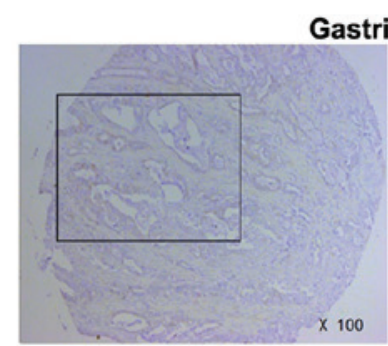

C
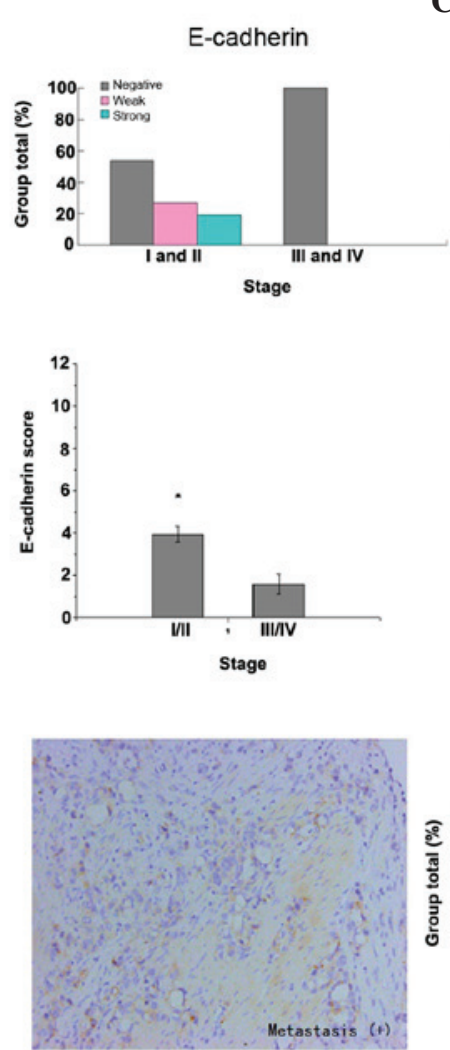
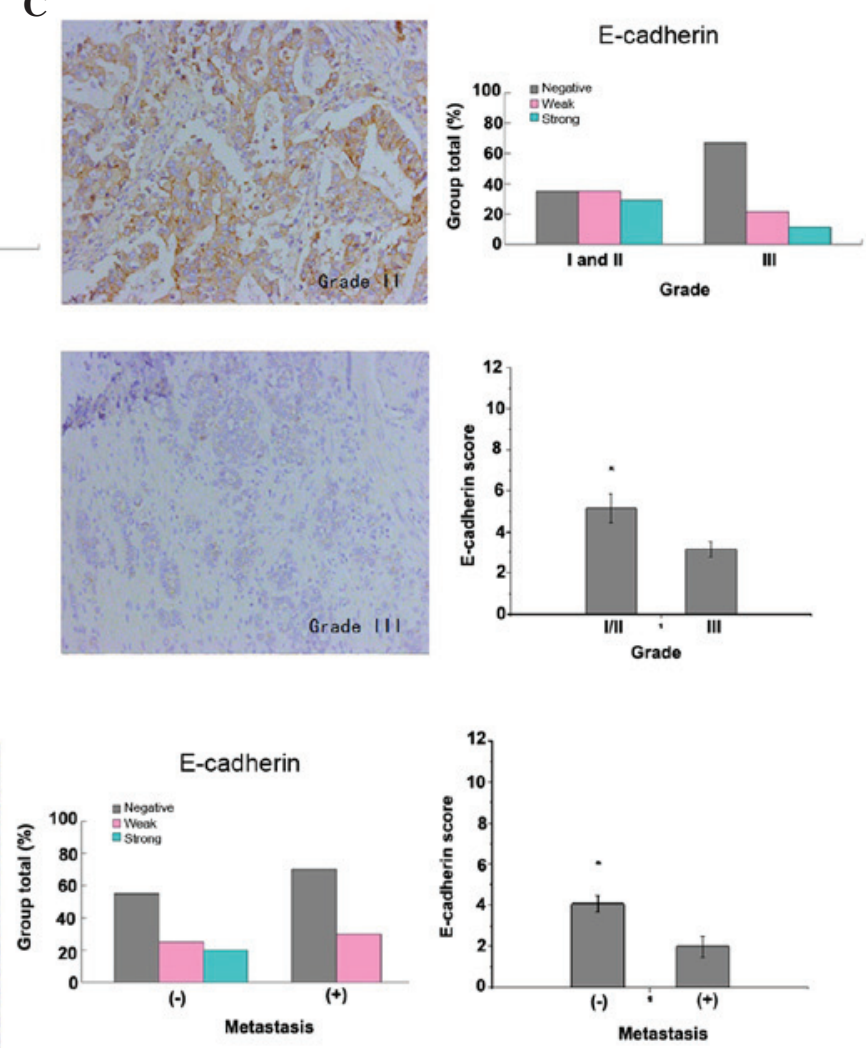

Figure 2. E-cadherin expression in samples of gastric cancer and its association with pathological features. (A) E-cadherin expression was detected by immunohistochemical analysis of tissue microarrays. Representative images of E-cadherin expression in normal gastric mucosa and gastric cancer tissue are shown (magnification, x100, and x200 for the inserts). The staining of E-cadherin in normal gastric mucosa cells was strongly positive, whereas in gastric cancer tissue cells, it was weakly positive or negative. (B) E-cadherin expression was negatively correlated with tumor stage (P=0.029 for stages I and II vs. stages III and IV). Representative images of stage I and III tumors are shown (magnification, x200). (C) E-cadherin expression was negatively correlated with tumor differentiation ( $\mathrm{P}=0.050$ for grades I and II vs. grade III). Representative images of grade II (strongly positive) and III (weakly positive) tumors are shown (magnification, $\mathrm{x} 200$ ). (D) E-cadherin expression was negatively correlated with lymph node (or distant) metastasis ( $\mathrm{P}=0.037$ for non-metastasis group vs. metastasis group). Representative images of gastric cancer with or without metastasis are shown (magnification, x200).

\section{Discussion}

The present study aimed to determine the clinical significance of FoxM1 and E-cadherin expression, and to uncover any critical correlation between FoxM1 and E-cadherin in driving the progression of gastric cancer. On the basis of the present results, there is a clear association between FoxM1, E-cadherin and clinicopathological features. Additionally, a negative correlation between FoxM1 expression and E-cadherin expression was identified in gastric cancer. Based on the aforementioned findings, it is possible to suggest that the dysregulated expression of FoxM1 may be responsible for E-cadherin expression, which may be a critical contributor to the EMT of gastric cancer. The negative correlation between FoxM1 and E-cadherin suggests that there may be a FoxM1/E-cadherin signaling pathway that ultimately results in the metastasis of gastric cancer.
FoxM1, a transcription factor belonging to the Fox protein superfamily, is involved in the complicated process of temporal and spatial gene expression during embryonic development and tissue homeostasis (17). Lines of newly-presented evidence have demonstrated that FoxM1 is a key regulator, not only playing a central role in controlling gene expression in both the G1-S and G2-M phases, but is also essential for mitotic progression and stability of chromosomes (17). Furthermore, FoxM1 has been implicated in different aspects of the progression of various cancers, including cell proliferation, tumor angiogenesis, migration, invasion, EMT and metastasis (18). According to the literature, the expression of FoxM1 is particularly high in pancreatic cancer (6), breast cancer (7), colorectal cancer (8) and hepatocellular carcinoma (12), as well as in a large number of other types of tumor cells (19), indicating that FoxM1 greatly contributes 
A

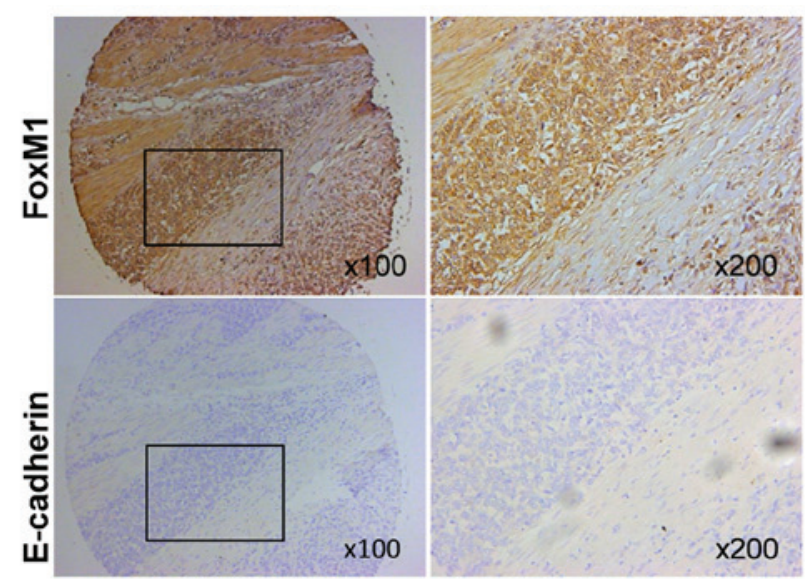

C

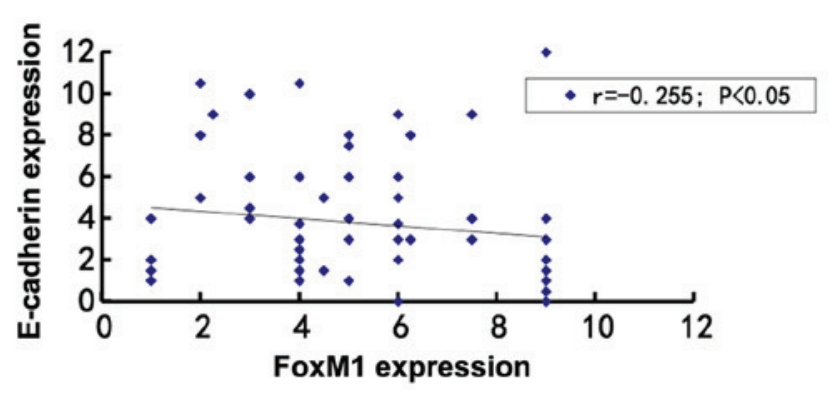

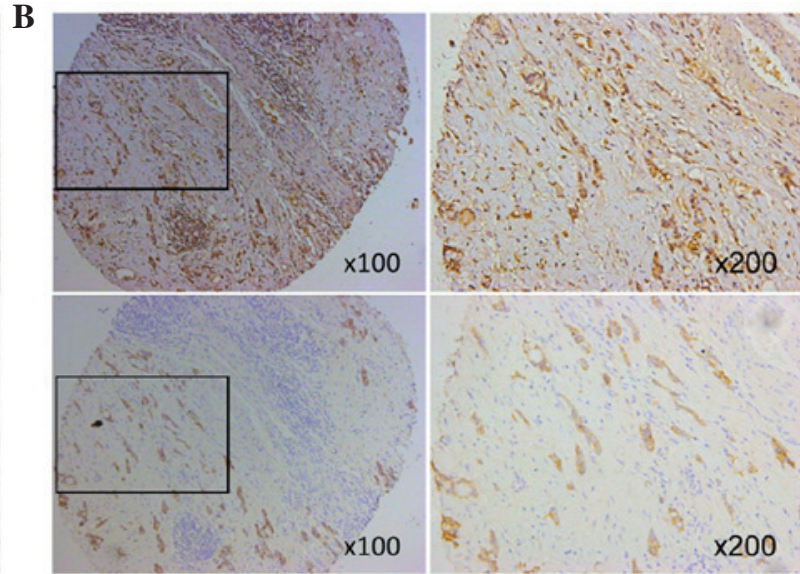

D

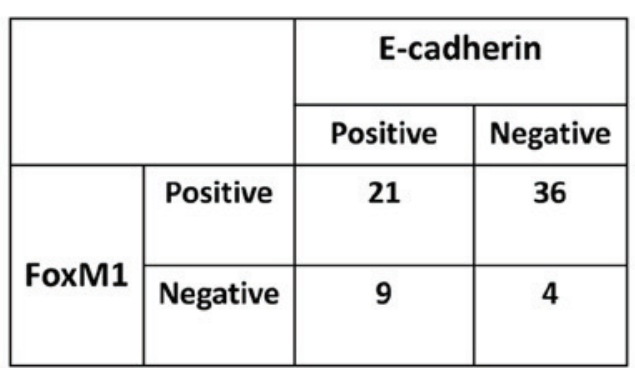

Figure 3. Co-expression of FoxM1 and E-cadherin expression in gastric cancer. (A) Immunohistochemical staining was performed with antibodies against FoxM1 and E-cadherin in gastric cancer tissue. Representative images of strongly-positive FoxM1 and negative E-cadherin staining are shown (magnification, x100, and x200 for the inserts). (B) Representative images of strong/moderate FoxM1 and moderate E-cadherin staining in gastric cancer sections are shown (magnification, x100, and x200). (C) A direct correlation between FoxM1 and E-cadherin expression in gastric cancer tissue was identified (n=70; Spearman's rank correlation test, $\mathrm{r}=-0.255 ; \mathrm{P}=0.035$ ). Of note, certain dots on the graph represent $>1$ sample (overlapped scores). (D) Summary of the expression of FoxM1 and E-cadherin in 70 gastric cancer samples. FoxM1, forkhead box M1.

to the development and progression of numerous human tumors. Clinical evidence reported by $\mathrm{Chu}$ et al (20) revealed that the expression of FoxM1 protein was much higher in colorectal cancer in comparison with that in the surrounding normal tissue. Additionally, high FoxM1 expression was closely correlated with advanced tumor-node-metastasis (TNM) stage, lymph node metastasis and low 5-year survival rate. In our previous studies, it was demonstrated that the high expression of FoxM1 in pancreatic cancer was significantly correlated with poor tumor differentiation, advanced TNM stage and lymph node metastasis, thus manifesting the key role of FoxM1 in the progression of pancreatic cancer (6). Similarly, the present study revealed that high expression of FoxM1 was strongly correlated with poor tumor differentiation, advanced tumor stage and lymph node (or distant) metastasis, thus revealing the critical role of FoxM1 in the development, invasion and metastasis of gastric cancer. Accumulating evidence suggests that FoxM1 plays a critical role in the migration, invasion and metastasis of different type of cancers, including pancreatic cancer (21), lung adenocarcinoma (22) and prostate cancer (23), by acquiring the EMT phenotype. Our previous study detected the expression of FoxM1 in different pancreatic cancer cell lines, and the expression of FoxM1 in pancreatic cancer cell lines with pronounced metastatic ability was observed to be higher compared with that in pancreatic cancer cell lines with poor metastatic ability (21). Kong et al demonstrated that reduced FoxM1 expression can reverse the mesenchymal phenotype of non-small cell lung cancer (NSCLC) cell lines into the epithelial phenotype (24). In addition, the migratory ability of NSCLC cell lines was decreased upon transfection with small interfering RNA targeting FoxM1 (24). Overall, the above observations further strengthen the hypothesis that FoxM1 may function as a tumor promoter in gastric cancer.

It is well known that E-cadherin, which is mainly expressed by epithelial cells and is responsible for cell-cell adhesion in tissues, plays a critical role in the maintenance of cell morphology and homeostasis (25). Several lines of evidence have demonstrated that E-cadherin, termed a tumor suppressor, inhibits the migration, invasion and dissemination of tumors by affecting the EMT, which is acknowledged as the key mechanism underlying metastasis of various tumor cells, including hepatocellular (26), breast (27) and colorectal cancer (28). Zhai et al (29) observed that decreased expression of E-cadherin was critically correlated with various clinicopathological features of hepatocellular cancer, including late disease stages, poor tumor differentiation and lymph node metastasis. Consistent with these observations, in the present study, reduced expression of E-cadherin was also strongly associated with advanced tumor stage, poor tumor differentiation and lymph node metastasis, indicating that E-cadherin detection may be a significant prognostic hallmark in gastric cancer. Based on the published literature, there are numerous potential mechanisms involved in migration, invasion and metastasis of tumors. For example, it was stated by Zhou et al that the activation of the estrogen receptor $\beta 1$ can upregulate the expression of E-cadherin in breast cancer and inhibit its migration and invasion (27). However, little is known concerning the regulatory mechanisms involved in invasion and 
metastasis and their association with FoxM1 and E-cadherin in gastric cancer. In the current study, a negative correlation was detected between FoxM1 and E-cadherin in tissue and cell lines of gastric cancer. Furthermore, a previous study by Wierstra (30) revealed that the transcription factor FoxM1c can directly combine with the promoter of the EMT-associated gene E-cadherin in mice and humans. Therefore, we hypothesize that FoxM1 can directly bind to the promoter of E-cadherin, thus inducing changes in EMT, which promotes invasion and metastasis in gastric cancer.

Taken together, the present findings have demonstrated the expression pattern of FoxM1 and E-cadherin in gastric cancer and adjacent normal tissue, and have also uncovered the correlation between FoxM1, E-cadherin and clinicopathological parameters in gastric cancer. The present results strongly suggest that FoxM1 and E-cadherin play a vital role in the development and progression of gastric cancer. The positive correlation between FoxM1 and E-cadherin in tissues and cell lines of gastric cancer indicate that there may be a FoxM1/E-cadherin signaling pathway contributing to the invasion and metastasis of gastric cancer, which may be a promising molecular target for the treatment of gastric cancer in the future.

\section{Acknowledgements}

The present study was partly supported by grant no. 13PJD024 (awarded to C.H.) from the Shanghai Municipal Human Resources and Social Security Bureau (Shanghai, China), grant no. XYQ2013092 (awarded to C.H.) from the Shanghai Health and Family Planning Commission (Shanghai, China) and grant no. 14411966800 (awarded to C.H.) from the Shanghai Municipal Science and Technology Commission (Shanghai, China).

\section{References}

1. Cho JY: Molecular diagnosis for personalized target therapy in gastric cancer. J Gastric Cancer 13: 129-135, 2013.

2. Nagini S: Carcinoma of the stomach: A review of epidemiology, pathogenesis, molecular genetics and chemoprevention. World $\mathbf{J}$ Gastrointest Oncol 4: 156-169, 2012.

3. Yatsuya H, Toyoshima H, Mizoue T, Kondo T, Tamakoshi K, Hori Y, Tokui N, Hoshiyama Y, Kikuchi S, Sakata K, et al: Family history and the risk of stomach cancer death in Japan: Differences by age and gender. Int J Cancer 97: 688-694, 2002.

4. Halasi M and Gartel AL: Targeting FOXM1 in cancer. Biochem Pharmacol 85: 644-652, 2013.

5. Alvarez-Fernández M and Medema RH: Novel functions of FoxM1: From molecular mechanisms to cancer therapy. Front Oncol 3: 30, 2013.

6. Huang C, Xie D, Cui J, Li Q, Gao Y and Xie K: FOXM1c promotes pancreatic cancer epithelial-to-mesenchymal transition and metastasis via upregulation of expression of the urokinase plasminogen activator system. Clin Cancer Res 20: 1477-1488, 2014.

7. Yang C, Chen H, Tan G, Gao W, Cheng L, Jiang X, Yu L and Tan Y: FOXM1 promotes the epithelial to mesenchymal transition by stimulating the transcription of Slug in human breast cancer. Cancer Lett 340: 104-112, 2013.

8. Li D, Wei P, Peng Z, Huang C, Tang H, Jia Z, Cui J, Le X, Huang S and Xie K: The critical role of dysregulated FOXM1-PLAUR signaling in human colon cancer progression and metastasis. Clin Cancer Res 19: 62-72, 2013.

9. Li X, Tang D, Yao Y, Qi W and Liang J: Clinical significance and positive correlation of FoxM1 and Her-2 expression in gastric cancer. Clin Exp Med 14: 447-455, 2014.
10. Li Q, Zhang N, Jia Z, Le X, Dai B, Wei D, Huang S, Tan D and Xie K: Critical role and regulation of transcription factor FoxM1 in human gastric cancer angiogenesis and progression. Cancer Res 69: 3501-3509, 2009.

11. Yu C, Chen L, Yie L, Wei L, Wen T, Liu Y and Chen H: Targeting FoxM1 inhibits proliferation, invasion and migration of nasopharyngeal carcinoma through the epithelialto-mesenchymal transition pathway. Oncol Rep 33: 2402-2410, 2015.

12. Meng FD, Wei JC, Qu K, Wang ZX, Wu QF, Tai MH, Liu HC, Zhang RY and Liu C: FoxM1 overexpression promotes epithelial-mesenchymal transition and metastasis of hepatocellular carcinoma. World J Gastroenterol 21: 196-213, 2015

13. Bhatt T, Rizvi A, Batta SP, Kataria S and Jamora C: Signaling and mechanical roles of E-cadherin. Cell Commun Adhes 20: 189-199, 2013.

14. Yang SW, Ping YF, Jiang YX, Luo X, Zhang X, Bian XW and Yu PW: ATG4A promotes tumor metastasis by inducing the epithelial-mesenchymal transition and stem-like properties in gastric cells. Oncotarget: Jun 4, 2016 (Epub ahead of print).

15. Yu H, Shen Y, Hong J, Xia Q, Zhou F and Liu X: The contribution of TGF- $\beta$ in Epithelial-Mesenchymal Transition (EMT): Down-regulation of E-cadherin via snail. Neoplasma 62: 1-15, 2015.

16. Markiewicz A, Wełnicka-Jaśkiewicz M, Seroczyńska B, Skokowski J, Majewska H, Szade J and Żaczek AJ: Epithelial-mesenchymal transition markers in lymph node metastases and primary breast tumors-relation to dissemination and proliferation. Am J Transl Res 6: 793-808, 2014.

17. Bella L, Zona S, Nestal de Moraes G and Lam EW: FOXM1: A key oncofoetal transcription factor in health and disease. Semin Cancer Biol 29: 32-39, 2014.

18. Huang C, Du J and Xie K: FOXM1 and its oncogenic signaling in pancreatic cancer pathogenesis. Biochim Biophys Acta 1845: 104-116, 2014.

19. Koo CY, Muir KW and Lam EW: FOXM1: From cancer initiation to progression and treatment. Biochim Biophys Acta 1819: 28-37, 2012.

20. Chu XY, Zhu ZM, Chen LB, Wang JH, Su QS, Yang JR, Lin Y, Xue LJ, Liu XB and Mo XB: FOXM1 expression correlates with tumor invasion and a poor prognosis of colorectal cancer. Acta Histochem 114: 755-762, 2012.

21. Huang C, Qiu Z, Wang L, Peng Z, Jia Z, Logsdon CD, Le X, Wei D, Huang $S$ and Xie K: A novel FoxM1-caveolin signaling pathway promotes pancreatic cancer invasion and metastasis. Cancer Res 72: 655-665, 2012.

22. Wei P, Zhang N, Wang Y, Li D, Wang L, Sun X, Shen C, Yang Y, Zhou $\mathrm{X}$ and Du X: FOXM1 promotes lung adenocarcinoma invasion and metastasis by upregulating SNAIL. Int J Biol Sci 11: 186-198, 2015.

23. Wang Y, Yao B, Wang Y, Zhang M, Fu S, Gao H, Peng R, Zhang L and Tang J: Increased FoxM1 expression is a target for metformin in the suppression of EMT in prostate cancer. Int $\mathrm{J}$ Mol Med 33: 1514-1522, 2014.

24. Kong FF, Zhu YL, Yuan HH, Wang JY, Zhao M, Gong XD, Liu F, Zhang WY, Wang CR and Jiang B: FOXM1 regulated by ERK pathway mediates TGF- $\beta 1$-induced EMT in NSCLC. Oncol Res 22: 29-37, 2014

25. van Roy F: Beyond E-cadherin: Roles of other cadherin superfamily members in cancer. Nat Rev Cancer 14: 121-134, 2014.

26. Nakagawa H, Hikiba Y, Hirata Y, Font-Burgada J, Sakamoto K, Hayakawa Y, Taniguchi K, Umemura A, Kinoshita H, Sakitani K, et al: Loss of liver E-cadherin induces sclerosing cholangitis and promotes carcinogenesis. Proc Natl Acad Sci USA 111: 1090-1095, 2014.

27. Zhou Y, Ming J, Xu Y, Zhang Y and Jiang J: ER $\beta 1$ inhibits the migration and invasion of breast cancer cells through upregulation of E-cadherin in a Id1-dependent manner. Biochem Biophys Res Commun 457: 141-147, 2015.

28. Tang W, Zhu Y, Gao J, Fu J, Liu C, Liu Y, Song C, Zhu S, Leng Y, Wang G, et al: MicroRNA-29a promotes colorectal cancer metastasis by regulating matrix metalloproteinase 2 and E-cadherin via KLF4. Br J Cancer 110: 450-458, 2014.

29. Zhai X, Zhu H, Wang W, Zhang S, Zhang Y and Mao G: Abnormal expression of EMT-related proteins, S100A4, vimentin and E-cadherin, is correlated with clinicopathological features and prognosis in HCC. Med Oncol 31: 970, 2014.

30. Wierstra I: The transcription factor FOXM1c binds to and transactivates the promoter of the tumor suppressor gene E-cadherin. Cell Cycle 10: 760-766, 2011 